\title{
Modelo numérico tridimensional para la simulación de procesos de tratamiento superficial de materiales con láser ${ }^{(\bullet)}$
}

\author{
A. García-Beltrán ${ }^{(* * *)}$ y J.L. Ocaña ${ }^{(* *, * * *)}$
}

\begin{abstract}
Resumen Se desarrolla un modelo numérico para la predicción y análisis del temple superficial de aceros por láser y de otros tratamientos superficiales de materiales metálicos y no metálicos. Partiendo de los datos del material y de su geometría, el modelo tridimensional de simulación desarrollado resuelve el problema de la transferencia de calor de la fuente generada por el haz láser dependiente del tiempo y de la temperatura y ha sido contrastado teórica y experimentalmente tanto para materiales metálicos como no metálicos. Ejemplos típicos de su aplicación son el temple superficial de acero al carbono y el análisis de los campos térmicos en materiales de baja conductividad térmica (como el vidrio óptico) en aplicaciones de pulido con láser.
\end{abstract}

Palabras clave: Láser. Tratamientos superficiales. Temple. Acero. Simulación numérica

\section{A 3D numerical model for the simulation of laser material surface treatment processes}

\begin{abstract}
A numerical model for the predictive assessment of laser transformation hardening of steels and other surface treatments both in metallic and non-metallic materials has been developed. Starting from material and geometry data, the problem of heat diffusion from the thermal source provided by the laser is solved with complete space and temperature-dependence. The developed 3D simulation model has been successfully checked against theoretical and experimental results both for metallic and non-metallic materials. Typical examples of its application have been the thermal surface hardening of steels and the analysis of thermal fields in low diffusivity materials (as optical glass) in laser polishing applications.
\end{abstract}

Keywords: Laser. Surface treatments. Transformation hardening. Steel. Numerical simulation.

\section{INTRODUCCIÓN}

Con vistas a la obtención de una herramienta de cálculo para la predicción del comportamiento de los materiales bajo una irradiación láser, se ha desarrollado un modelo numérico para la predicción de los campos térmicos dependientes del tiempo

$(\bullet \quad$ Trabajo recibido el día 30 de junio de 1998 y aceptado en su forma final el día 25 de marzo de 1999.

(*) Dpto. de Automática, Ing. Electrónica e Informática Industrial. ETSI Industriales. C/ José Gutiérrez Abascal, 2. E-28006 Madrid (España).

(**) ETSIIMLAS. Dpto. de Física Aplicada. ETSI Industriales C/ José Gutiérrez Abascal, 2. E-28006 Madrid (España).

(***) Escuela Politécnica Superior. Universidad de La Coruña. C/ Mendizábal s/n. E-15403 Ferrol (La Coruña, España). durante los procesos correspondientes con consideración explícita de las propiedades ópticas y termofísicas de los materiales dependientes de la temperatura.

Desde un punto de vista general, los modelos matemáticos para la predicción de campos térmicos pueden dividirse en modelos analíticos y numéricos. Rappaz (1) realiza un estudio general de las ecuaciones de partida y diferentes clases de métodos (en especial numéricos) utilizados para su resolución. Ion (2) concluye que la adopción de un método determinado dependerá de las necesidades reales de predicción y las disponibilidades de recursos computacionales. De acuerdo con Ames (3) y Lapidus y Pinder (4), los métodos que emplean modelos numéricos son, en general, los más adecuados para la simulación numérica de problemas 
físicos como la transferencia de calor, en los que los valores de los campos vectoriales pueden presentar grandes gradientes en geometrías complicadas.

Siguiendo esta recomendación, se han desarrollado códigos de simulación numéricos muy útiles para la resolución de diferentes problemas en el campo de la interacción láser-materia a moderadas intensidades que permiten la transformación o cambios de fase de materiales. Mazumder y Steen (5) desarrollan un modelo 3D de elementos finitos cuasiestacionario para la predicción de procesos de materiales con láser, con consideración de propiedades de los materiales dependientes de la temperatura y pérdidas por convección y radiación. Fritsch y Bergmann (6) han desarrollado un modelo combinado 2D-3D de elementos finitos para el estudio de la difusión del carbón durante las transformaciones en procesos de tratamiento térmico de aceros con láser. Kechemair (7) desarrolla un modelo bidimensional de elementos finitos para el análisis de procesos de temple superficial, con propiedades térmicas y ópticas de los materiales dependientes de la temperatura, y compara con éxito sus resultados numéricos con pruebas experimentales de temple superficial de acero 35NCD16. Galantucci y Tricarico (8) proponen dos modelos para procesos estacionarios y transitorios de tratamientos térmicos con láser, respectivamente, basados en el empleo del código comercial de elementos finitos ANSYS que, sin embargo, carece de una cierta aproximación al problema real debido a la no consideración de la variación de las propiedades ópticas y térmicas de los materiales con la temperatura.

Tratando de establecer una solución comprensible del problema físico del calentamiento superficial de materiales con láser con un tratamiento de elementos finitos, pero teniendo en cuenta los aspectos más importantes $\mathrm{y}$, a veces, más complejos de la interacción láser-materia a intensidades típicas de interés, se ha desarrollado un modelo de cálculo denominado TEMPLUM, capaz de determinar la evolución de los campos térmicos y las propiedades físicas resultantes de probetas de materiales metálicos y no metálicos sujetas a procesos de tratamiento superficial y con una resolución espacial limitada tan sólo por la disponibilidad de recursos computacionales. El código desarrollado ha sido contrastado con éxito frente a resultados experimentales en configuraciones de referencia típicas y está siendo empleado actualmente en la predicción de campos térmicos en procesos de tratamiento térmico superficial de aceros y de pulido de vidrio óptico.

\section{CARACTERÍSTICAS DEL CÓDIGO}

El código desarrollado TEMPLUM (8) calcula la solución de la ecuación de transferencia del calor en problemas de tratamiento superficial con láser con consideración específica de los fenómenos físicos particulares que entran en juego en este tipo de procesos. Se ha demostrado que la modificación durante el tratamiento láser de las propiedades ópticas y termofísicas de los materiales influye decisivamente en los resultados finales del proceso y este comportamiento no lineal debe ser tenido en cuenta al mismo tiempo que las características espaciotemporales que caracterizan a los códigos de simulación numérica usuales.

El modelo se ha construido partiendo de la base de un código de elementos finitos de propósito general, TOPAZ (9), desarrollado para el estudio de la solución de problemas de transmisión del calor y electromagnéticos $\mathrm{y}$, de su versión tridimensional, TOPAZ3D (10), junto con las incorporaciones mencionadas de las características físicas particulares concernientes a los procesos de tratamiento térmico superficial de materiales con láser.

Como primera característica a resaltar se ha llevado a cabo el tratamiento apropiado de las condiciones de incidencia sobre la probeta de material, con la consideración específica de la distribución de intensidad transversal del haz láser, la absorción de intensidad de la superficie del material y su variación en función de la temperatura durante el proceso, el factor de absorción o atenuación de la intensidad de radiación del material y la variación de las propiedades térmicas como consecuencia de los ciclos térmicos inducidos durante los procesos.

En un trabajo previo, Kechemair (7) consideró las posibles variaciones de la absortancia de la superficie debido al efecto de la interacción lásermateria con la probeta y tuvo en cuenta la definición de una cierta temperatura media de proceso con el fin de plantearse la variación, en base a las temperaturas, de las propiedades térmicas del material tratado (acero 35NCD16, en ese caso particular). Sin embargo, de acuerdo con sus propias conclusiones y los resultados obtenidos considerando totalmente estas variaciones, la solución precisa del problema sólo puede ser obtenida en el caso general de una dependencia completa de la temperatura.

Por otro lado, surgen importantes incertidumbres desde el punto de vista práctico cuando se determina la efectividad real de un tratamiento superficial debido a una consideración inadecuada de la distribución de energía transversal del haz láser. Como práctica común, los códigos de simulación que abordan el tratamiento superficial con láser consideran una distribución de intensidad constante sobre la superficie de incidencia (perfil uniforme como idealización del resultado de homogeneización del haz con sistemas caleidoscópicos) o un modo gaussiano dado, típicamente un modo $\mathrm{TEM}_{00}$. Sin embargo, en el caso general, la distribución de intensidad transversal del haz incidente 
varía sustancialmente de cualquiera de los casos anteriores y normalmente sólo puede ser reconstruido mediante una serie de Fourier de términos no triviales que incluyen varios de los modos gaussianos de menor orden (11).

Con el objetivo de realizar una aproximación realista al problema general del tratamiento con láser de materiales en fase condensada, es decir, sin consideración de la formación de vapor o plasma, típica de los tratamientos superficiales con láser desde el punto de vista estricto de una predicción numérica de campos térmicos sin movimiento de fluidos, pero tratando de considerar de forma adecuada tanto los efectos físicos de los cambios en las propiedades ópticas y termofísicas de los materiales como la geometría del haz incidente, el código TEMPLUM incluye, como características más importantes, módulos de preprocesado y rutinas que resuelven los problemas referidos anteriormente y facilitan, desde el punto de vista numérico (inicialmente, sin considerar interpretaciones complejas que puedan derivarse de las transformaciones del material), un alto grado de versatilidad y fiabilidad en función de la adecuación de las constantes de los materiales especificadas.

TEMPLUM es un código computacional dependiente del tiempo, que resuelve la ecuación de la transferencia del calor en geometrías cartesianas rectangulares:

$$
\rho C_{\mathrm{p}} \frac{\partial T}{\partial t}-\nabla \cdot(\overline{\bar{\kappa}} \nabla T)=E(r, t)
$$

que puede desarrollarse como

$$
\begin{gathered}
\rho C_{\mathrm{p}} \frac{\partial T}{\partial t}=\frac{\partial}{\partial x}\left(\kappa_{\mathrm{xx}} \frac{\partial T}{\partial \mathrm{x}}\right)+\frac{\partial}{\partial \mathrm{y}}\left(\kappa_{\mathrm{yy}} \frac{\partial T}{\partial \mathrm{y}}\right)+\frac{\partial}{\partial \mathrm{z}}\left(\kappa_{\mathrm{zz}} \frac{\partial T}{\partial \mathrm{z}}\right)+ \\
+A(\mathrm{x}, \mathrm{y}, \mathrm{z}, t)=\nabla \cdot(\kappa \nabla T)+E(\mathrm{x}, \mathrm{y}, \mathrm{z}, t)
\end{gathered}
$$

capaz de tratar separadamente los problemas bidimensionales y los tridimensionales empleando el método de los elementos finitos para la discretización espacial y un esquema de Crank-Nicholson semiimplícito para la variable temporal. El código ofrece convergencia, estabilidad, precisión y una sensibilidad típica de los métodos numéricos empleados (8-11), tomando como parámetros de entrada las propiedades térmicas y ópticas de los materiales, la geometría de la pieza, las condiciones de proceso y los parámetros de simulación elegidos. Como datos de salida facilita, en cualquier instante de tiempo, la intensidad de energía absorbida en cada nodo de la malla modelizada del material, las temperaturas y velocidades de calentamiento-enfriamiento, los flujos de intensidad en cada nodo y las temperaturas máximas y mínimas en los nodos de la malla (9).

\section{RESULTADOS DEL MODELO}

El código TEMPLUM ha sido empleado para la simulación de diferentes tipos de problemas en el campo de las aplicaciones de tratamiento superficial de materiales con láser. Se presentan dos casos seleccionados como ejemplos de los tipos de resultados de la simulación que el código facilita.

\subsection{Tratamiento superficial con láser de $\mathrm{CO}_{2}$ de un acero al carbono F1140}

En este ejemplo se presentan las posibilidades estándar del código como herramienta de predicción del temple superficial de aceros (la primera y más típica aplicación del código desde sus orígenes). Para el desarrollo de esta aplicación se emplea la geometría mostrada en la figura 1.

El haz láser procedente de un resonador óptico que produce un haz de potencia constante en el intervalo de 1.300 a $1.700 \mathrm{~W}$ incide sobre la superficie de una pieza de $100 \times 20 \times 5 \mathrm{~mm}^{3}$ con los parámetros dados en la tabla I.

El objetivo del proceso considerado es la transformación de la microestructura de la superficie de la pieza y consecuentemente, la modificación de las propiedades mecánicas. De acuerdo con esto, un elemento fundamental para la evaluación de los resultados del código es el análisis de las zonas en las que las propiedades mecánicas han sufrido un cambio como consecuencia del ciclo térmico producido por el haz láser. Sobre la base de este procedimiento, se lleva a cabo una evaluación indirecta, a la vez que precisa, de la historia térmica de los puntos de la probeta que indican claramente la extensión de los campos térmicos en función de las temperaturas críticas de las transformaciones de los aceros. En el caso del acero analizado con $\mathrm{Ac}_{1}=$ $790{ }^{\circ} \mathrm{C}$ y $\mathrm{Ac}_{3}=915{ }^{\circ} \mathrm{C}$ y las propiedades ópticas y termofísicas indicadas en la tabla II, y con el fin de

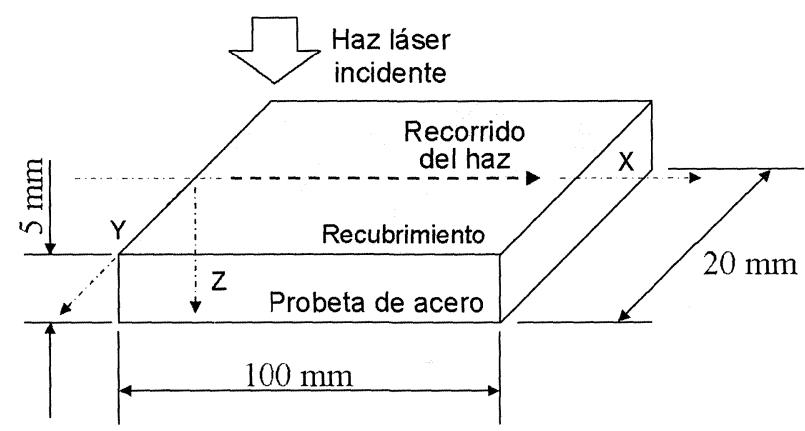

FIG. 1.- Esquema experimental del tratamiento térmico con láser de $\mathrm{CO}_{2}$ de un acero $\mathrm{F} 1140$

FIG. 1.- Experimental scheme of a F1140 steel $\mathrm{CO}_{2}$ laser thermal treatment. 
TABLA I.- Parámetros de incidencia del haz considerado en el tratamiento térmico de acero F1140 con un láser de $\mathrm{CO}_{2}$

TABLE I.- Laser beam incident parameters for the F1140 steel $\mathrm{CO}_{2}$ laser thermal treatment

\begin{tabular}{|c|c|}
\hline Parámetros del haz & $\begin{array}{l}\text { Valores } \\
\text { (unidades) }\end{array}$ \\
\hline Potencia & $1.300 \pm 2 \%(\mathrm{~W})$ \\
\hline Longitud de onda & $10,6(\mu \mathrm{m})$ \\
\hline Diámetro característico & $4(\mathrm{~mm})$ \\
\hline Divergencia & $\leq 1,5(\mathrm{mrad})$ \\
\hline Intensidad media & $\approx 10^{4}\left(\mathrm{Wcm}^{-2}\right)$ \\
\hline $\begin{array}{l}\text { Modo de distribución de } \\
\text { intensidad transversal }\end{array}$ & Gaussiano TEM $_{01 *}$ \\
\hline
\end{tabular}

TABLA II.- Propiedades del acero F1140 empleado en el proceso analizado

TABLE II.- F1140 steel properties for the analysed process

\begin{tabular}{|c|c|}
\hline Propiedad (unidades) & Valor \\
\hline Absortividad $\left(\mathrm{m}^{-1}\right)$ & $\approx \infty(\lambda=10,6 \mu \mathrm{m})$ \\
Absortancia $($ Adim. $)$ & $\mathrm{f}(T)$, según figura 2 \\
Densidad $\left(\mathrm{kg} \cdot \mathrm{m}^{-3}\right)$ & $7,86 \cdot 10^{3}$ \\
Calor específico $\left(\mathrm{J} \cdot \mathrm{kg}^{-1 .}{ }^{\circ} \mathrm{C}^{-1}\right)$ & $\mathrm{f}(T)$, según figura 2 \\
Conductividad térmica & $\mathrm{f}(T)$, según figura 2 \\
$\left(\mathrm{W} \cdot \mathrm{m}^{\left.-1 .{ }^{\circ} \mathrm{C}^{-1}\right)}\right.$ & \\
\hline
\end{tabular}

mejorar los valores de la absortancia de la superficie del material, ésta se ha lijado y se ha recubierto de una fina capa de pintura negra pulverizada.

Para la predicción en las simulaciones numéricas de las zonas que han sufrido realmente las transformaciones metalúrgicas también se considera la influencia de la velocidad de calentamientoenfriamiento. En la figura 3 se muestran esquemáticamente las diferentes zonas de interés en el proceso.

Como ejemplo de los resultados del modelo se muestran en las figuras 4 y 5 los ciclos térmicos y velocidades de calentamiento-enfriamiento de puntos de la probeta modelizada pertenecientes al plano de simetría de un proceso realizado con un haz láser de $1.300 \mathrm{~W}$.

En la figura 6 se muestran las curvas isotermas una vez alcanzado el régimen estacionario, indicándose las zonas en las que se predice la transformación en el acero debido al ciclo térmico, que se
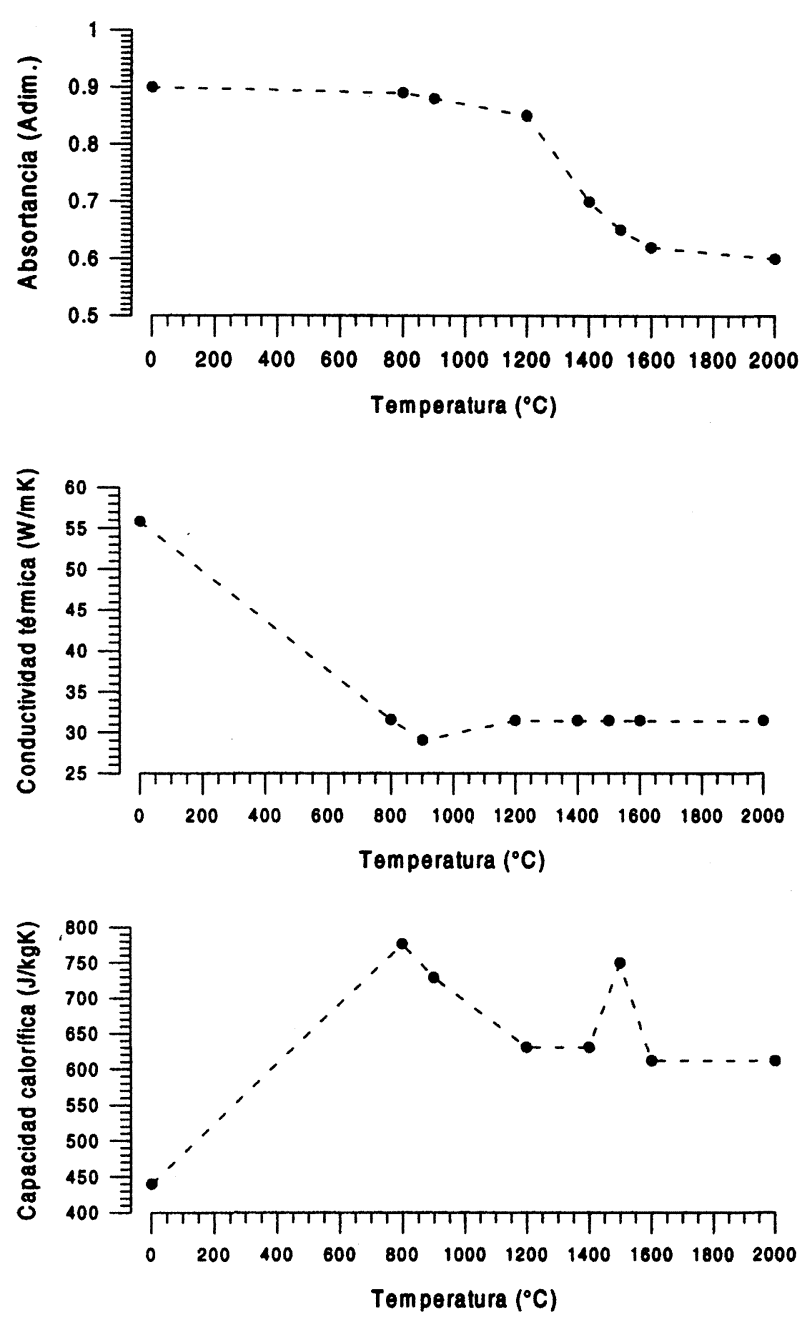

Fig. 2.- Absortancia de la superficie, conductividad térmica y capacidad calorífica del material en función de la temperatura

FIG. 2.- Temperature dependence of F1140 steel properties: absorptivity, thermal conductivity and heat capacity.

ajustan de forma muy sensible a los resultados obtenidos experimentalmente.

\subsection{Tratamiento superficial con láser de vidrio óptico B-270 con un haz láser de $\mathrm{CO}_{2}$ conformado}

Se lleva a cabo la simulación de un procedimiento real en el campo del pulido de vidrio óptico con láser, con el objetivo de mostrar, desde un punto de vista teórico, las posibles diferencias entre los efectos térmicos inducidos en materiales con patrones de intensidad uniformes y no uniformes y como prueba de las capacidades de simulación del modelo descrito.

En concreto, se realiza un análisis comparativo entre los efectos térmicos inducidos en el vidrio 


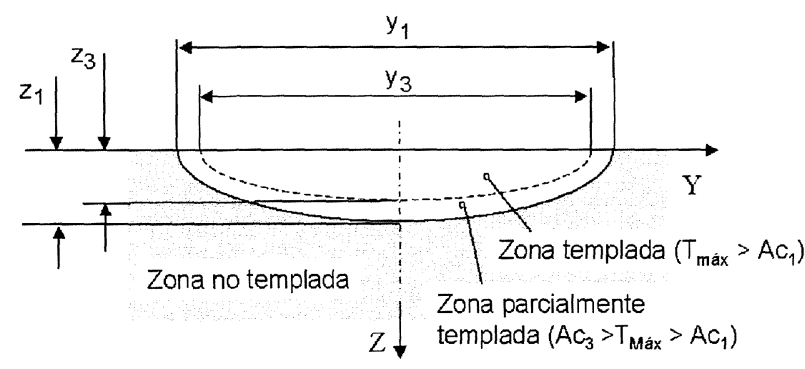

FIG. 3.- Esquema gráfico de las zonas transformadas en el proceso de temple superficial de un acero al carbono

FIG. 3.- Graphic scheme of the termal affected zones in a steel surface hardening process.

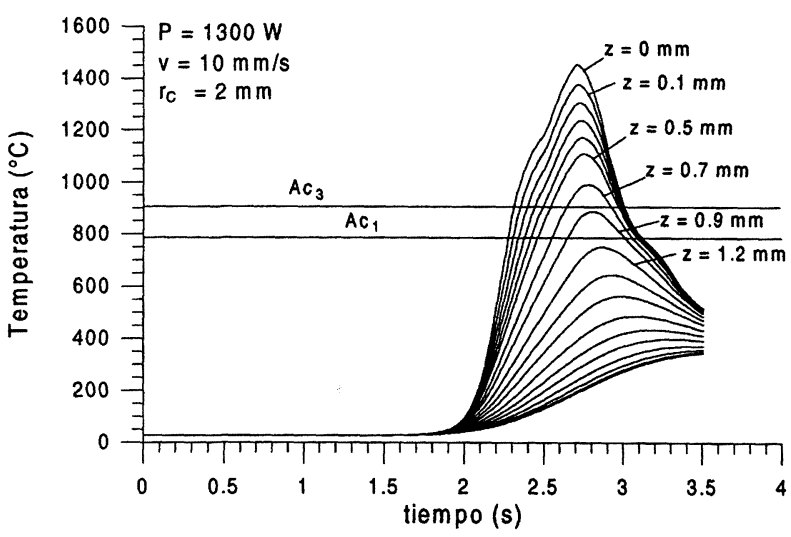

FIG. 4.- Ciclos térmicos de diferentes nodos situados a distinta profundidad en la probeta tratada con un haz láser de $\mathrm{CO}_{2} \mathrm{TEM}_{01 * \mathrm{y}} 1.300 \mathrm{~W}$

FIG. 4.- Material thermal cycles at different depth nodes obtained in a steel plate upon a $1300 \mathrm{~W}$ $\mathrm{TEM}_{\mathrm{Ol}} \mathrm{CO}_{2}$ laser irradiation.

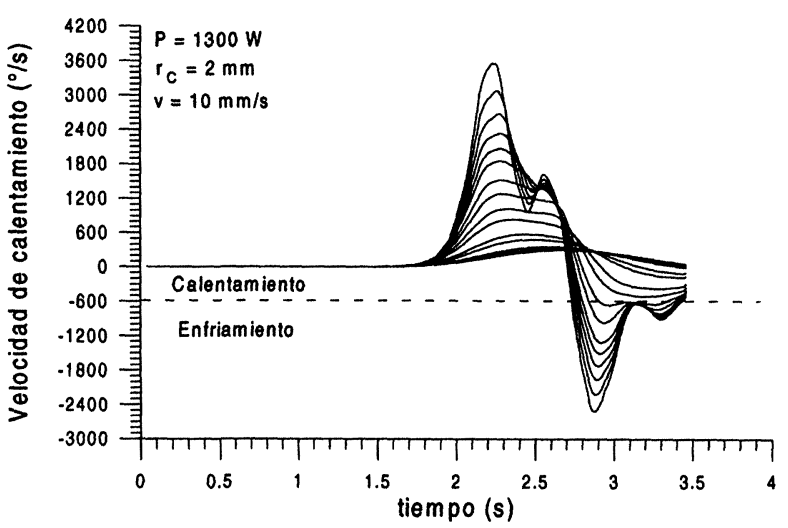

FIG. 5.- Velocidades de calentamiento-enfriamiento de diferentes nodos situados a distinta profundidad en la probeta tratada con un haz láser de $\mathrm{CO}_{2}$ $\mathrm{TEM}_{01 *}$ y $1.300 \mathrm{~W}$

FIG. 5.- Material heating/cooling rates at different depths obtained upon laser irradiation in a sample irradiated with a $1.300 \mathrm{WCO}_{2}$ laser beam (TEM $01 *$ mode).
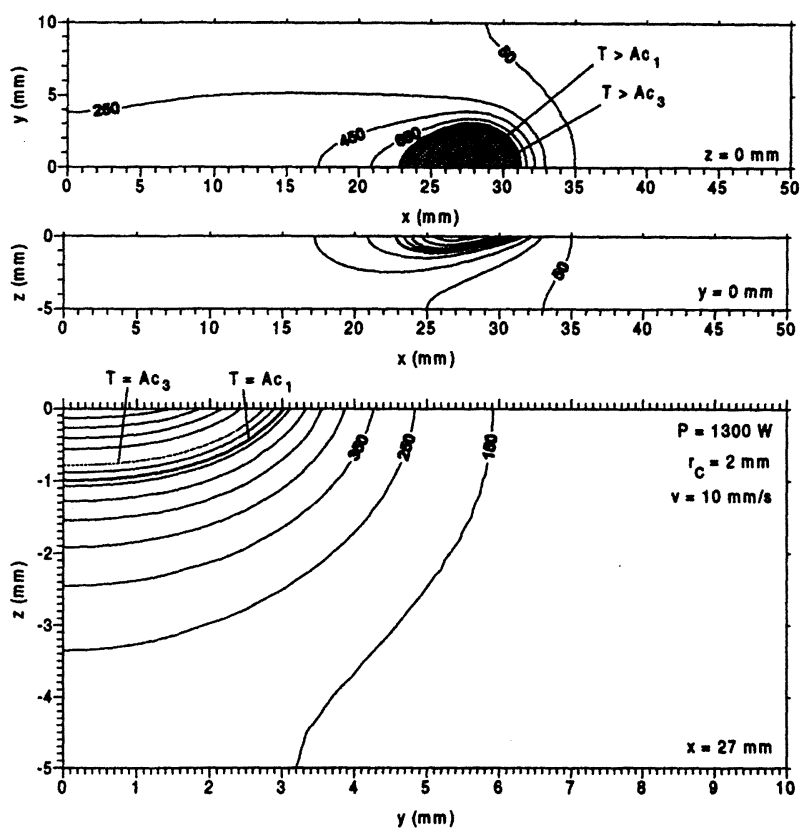

Fig. 6.- Predicción de los campos de temperatura una vez alcanzado el régimen estacionario en el tratamiento de la pieza de acero F1140 con un haz láser de $\mathrm{CO}_{2} \mathrm{TEM}_{01} *$ y $1.300 \mathrm{~W}$

FIG. 6.- Predicted stationary thermal contours for a F1140 steel sample irradiated with a $1300 \mathrm{~W}$ $\mathrm{CO}_{2}$ laser beam (TEM $01 *$ mode).

óptico por distribuciones de intensidad láser uniforme y no uniforme, resultantes de la conformación óptica de haces mediante espejos facetados (12).

Para esta comparación se ha considerado una probeta de vidrio óptico B-270 de $5 \mathrm{~mm}$ de espesor con las propiedades dadas en la tabla III y precalentado a $550^{\circ} \mathrm{C}$. Los patrones de intensidad considerados tienen respectivamente una potencia $\mathrm{P}_{0}=$ $58 \mathrm{~W}$ extendida uniformemente en una región cuadrada $|x|<1 / 2,|y|<1 / 2 y$, en el segundo caso, una distribución no uniforme definida según la siguiente expresión analítica:

$$
\begin{gathered}
I(x, y)=I_{0} \operatorname{sen}^{8}\left(\frac{2 \pi}{d} x\right) \operatorname{sen}^{8}\left(\frac{2 \pi}{d} y\right) \\
\text { para }|x| \leq 1 / 2 ;|y| \leq 1 / 2 \\
\text { o para }|x|>1 / 2 ;|y|>1 / 2
\end{gathered}
$$

con: $I_{0}=\frac{P_{\mathrm{o}}}{(35 / 128)^{2} l^{2}} ; d=1,2 \mathrm{~mm} ; l=8,4 \mathrm{~mm}$

representada gráficamente en la figura 7 y extendida sobre la misma área, de forma que la intensidad media de ambas distribuciones coinciden.

En las figuras 8 y 9 se muestran los campos térmicos resultantes en la superficie de incidencia para un tiempo característico de 1,5 s en ambos casos. 
TABLA III.- Propiedades del vidrio óptico B-270

TABLE III.- B-270 optical glass material and optical properties

\begin{tabular}{|c|c|}
\hline Propiedad (unidades) & Valor \\
\hline Absortividad $\left(\mathrm{m}^{-1}\right)$ & $7,10 \cdot 10^{4}(\lambda=10,6 \mu \mathrm{m})$ \\
Absortancia $($ Adim. $)$ & 1 \\
Densidad $\left(\mathrm{kg} \cdot \mathrm{m}^{-3}\right)$ & $2,5 \cdot 10^{3}$ \\
Calor específico $\left(\mathrm{J} \cdot \mathrm{kg}^{\left.-1 .{ }^{\circ} \mathrm{C}-1\right)}\right.$ & $10^{3}$ \\
Conductividad térmica & $1.047+0,001489 . \mathrm{T}$ \\
$\left(\mathrm{W} \cdot \mathrm{m}^{\left.-1 .{ }^{\circ} \mathrm{C}^{-1}\right)}\right.$ & $\left(\mathrm{T}<900{ }^{\circ} \mathrm{C}\right)$ \\
& $2.387\left(\mathrm{~T}>900{ }^{\circ} \mathrm{C}\right)$ \\
\hline
\end{tabular}

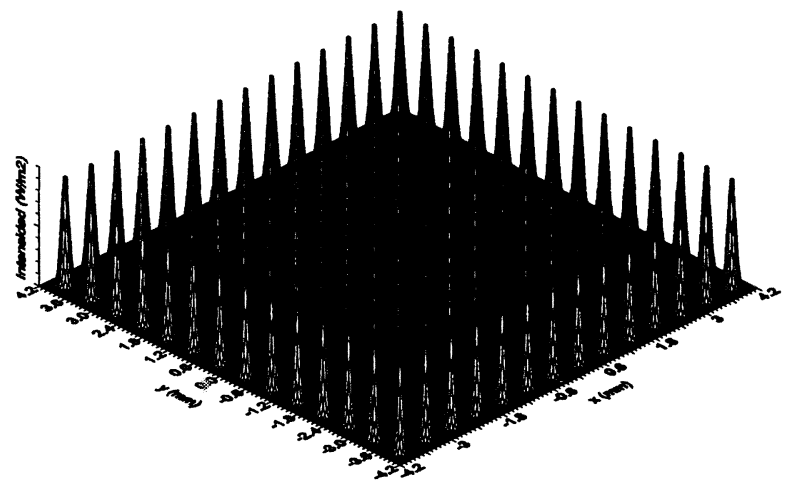

FIG. 7.- Representación gráfica tridimensional de la distribución transversal de intensidad utilizada en el proceso de vidrio óptico B270

FIG. 7.- Incoming laser beam intensity tridimensional pattern for the B270 optical glass process.

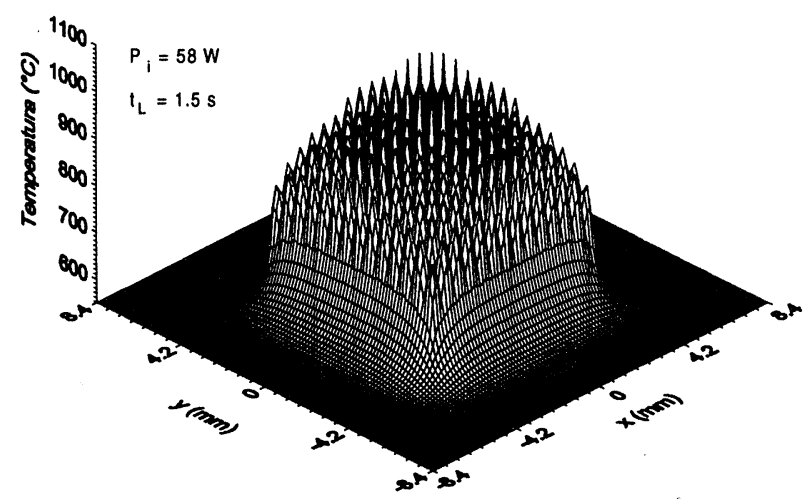

Fig. 8.- Campo térmico superficial resultante en la probeta de vidrio óptico B-270 con un haz láser de distribución no uniforme

FIG. 8.-B-270 optical glass thermal fields prediction following a regularly spiked laser intensity pattern irradiation.

Con la simple inspección visual de ambas figuras pueden observarse diferencias significativas

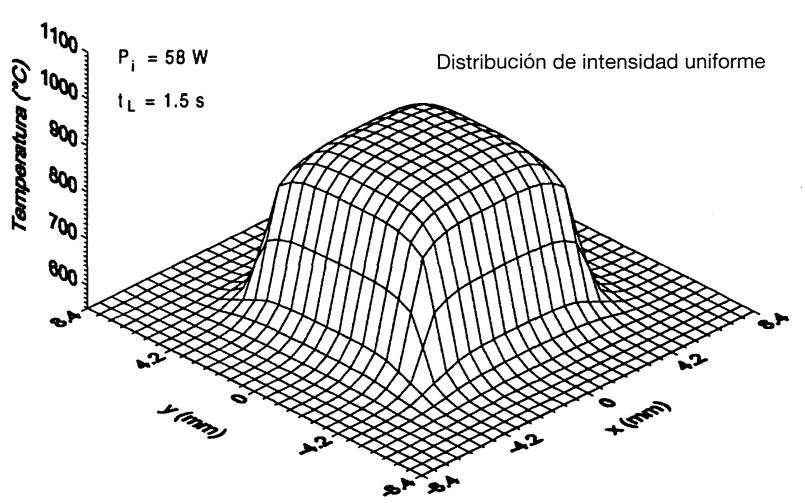

FIG. 9.- Campo térmico superficial resultante en la probeta de vidrio óptico B-270 con un haz láser de distribución uniforme

FIG. 9.-B-270 optical glass thermal fields prediction following a uniform intensity pattern irradiation.

entre las condiciones térmicas alcanzadas en cada caso. Los valores de las temperaturas máximas obtenidos en ambas piezas son sensiblemente diferentes: $900{ }^{\circ} \mathrm{C}$ en el caso de la distribución uniforme y $1.060^{\circ} \mathrm{C}$ en el caso del patrón de intensidad no uniforme. También los gradientes de temperatura inducidos en el material son muy diferentes tanto en el sentido transversal (perpendicular a la dirección de incidencia) como en la profundidad (perpendicular a la superficie).

Desde un punto de vista computacional, el caso analizado muestra un ejemplo muy representativo de las capacidades del modelo para la simulación de procesos de tratamiento con láser, de materiales no sólo metálicos, con un coeficiente de atenuación de la radiación láser muy elevado y una buena conductividad térmica, sino también con materiales de baja conductividad térmica en los que la radiación tiene una penetración considerable (como en el caso del vidrio óptico considerado) y en los que los gradientes térmicos pueden ser relativamente elevados y pueden causar dificultades en los esquemas de discretización de los modelos numéricos (13).

Con estos ejemplos de aplicación ha quedado demostrado el uso del código TEMPLUM utilizado, con los adecuados parámetros de discretización para asegurar la convergencia, la estabilidad y la precisión apropiadas además de unas condiciones de entorno cuidadosamente consideradas, y su valor como herramienta muy consistente y robusta para la simulación de tratamientos con láser de materiales en fase condensada, incluso en casos en los que otras herramientas de simulación similares han encontrado grandes dificultades. 


\section{ANÁLISIS EXPERIMENTAL}

\subsection{Tratamiento superficial con láser de $\mathrm{CO}_{2}$ del acero al carbono F1140}

En la figura 10, se muestra un corte metalográfico de la pieza modelizada en la simulación del punto 3.1 , junto con el correspondiente mapa térmico resultante del análisis numérico (Fig. 11).

El acuerdo entre los resultados teóricos y los experimentales es muy bueno y puede ser cuantificado a partir del perfil de microdurezas (ensayo Vickers) mostrado en la figura 12 en la que la zona transformada (templada) completamente y la zona de transición están claramente definidas (junto con una pequeña zona descarburada cerca de la superficie visible en la figura 13).

Dentro de los márgenes de error permisibles en las medidas, en la tabla IV se muestran los de la comparación de los resultados experimentales y numéricos.

\subsection{Tratamiento superficial de vidrio óptico B-270 con un haz láser conformado}

Como se reconoce en la sección anterior, y desde el punto de visto físico, el problema del pulido de vidrio óptico empleando haces láser conformados aunque no está directamente relacionado con el tratamiento térmico superficial de metales tiene que ser considerado como una clase decisiva de experimentos capaces de plantear los problemas más complejos para una herramienta de simulación como la descrita. De hecho, la perspectiva inicial del análisis de este tipo de tratamientos vino como

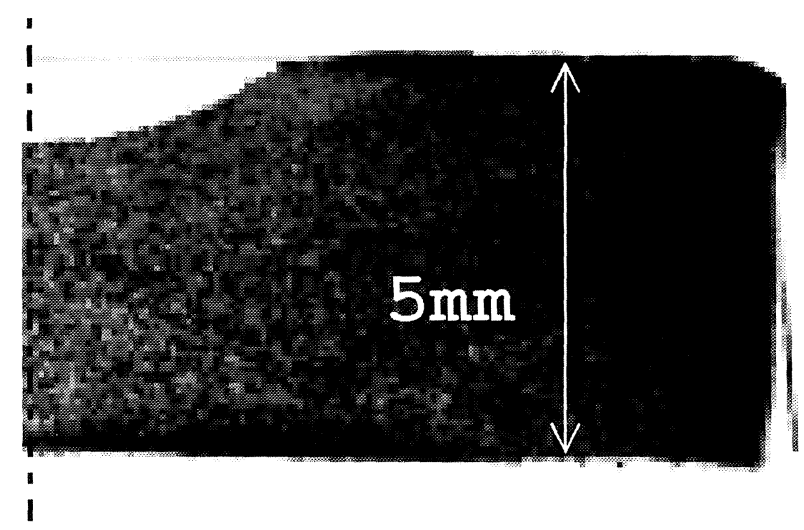

FIG. 10.- Corte metalográfico de la pieza tratada con un haz láser de $\mathrm{CO}_{2} \mathrm{TEM}_{01 *}$ de $1.300 \mathrm{~W}$ de potencia

FIG. 10.- Metallographic cross section of laser hardened F1140 steel sample irradiated with a $1.300 \mathrm{~W} \mathrm{CO}_{2}$ laser beam (TEM ${ }_{01 *}$ mode).

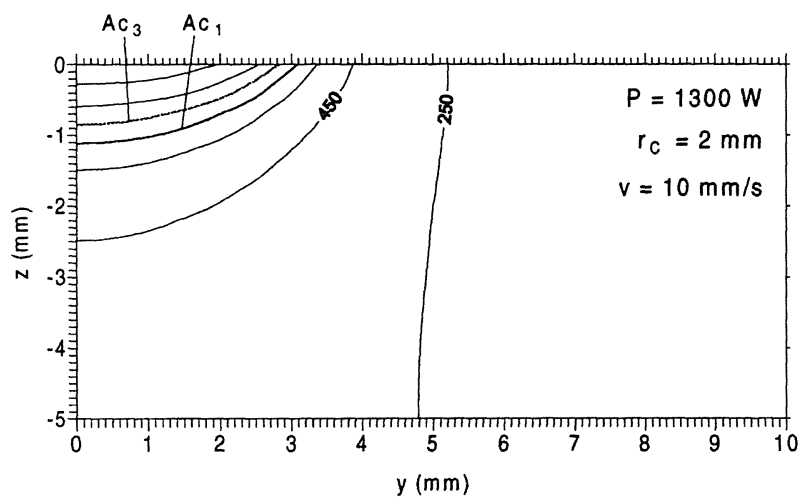

FIG. 11.- Mapa de temperaturas máximas alcanzadas en la pieza modelizada mediante el código TEMPLUM

FIG. 11.- Predicted maximum temperature contours of a F1140 steel sample model by TEMPLUM.

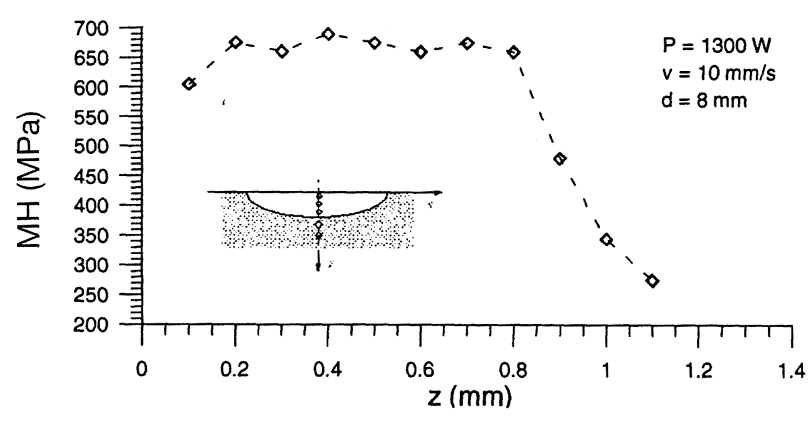

FIG. 12.- Perfil de microdurezas en función de la profundidad de la pieza tratada con un haz láser de $\mathrm{CO}_{2} \mathrm{TEM}_{01 *}$ y $1.300 \mathrm{~W}$

FIG. 12.- Microhardness profile of a laser hardened F1140 steel specimen irradiated with a $1.300 \mathrm{~W} \mathrm{CO}_{2}$ laser beam $\left(\right.$ TEM $_{01 *}$ mode) .

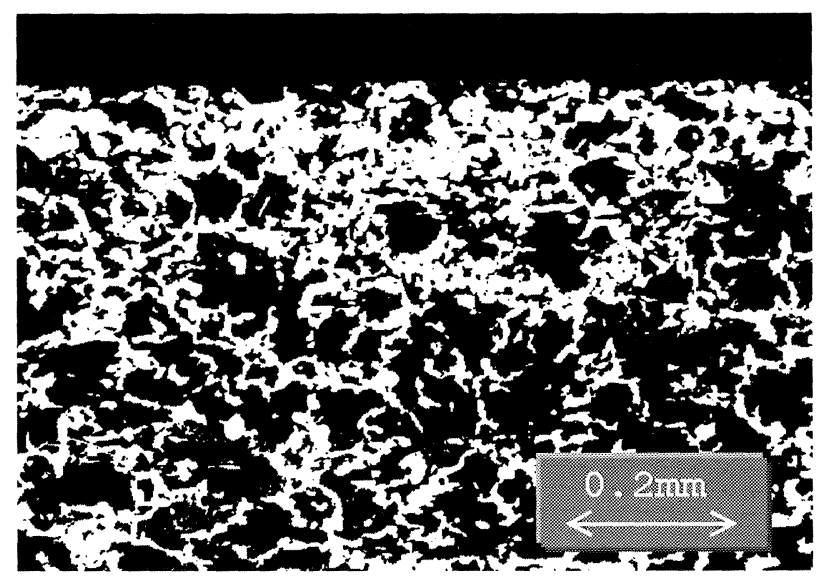

FIG. 13.- Micrografía de la superficie de la pieza tratada que muestra una pequeña capa descarburada

FIG. 13.- Micrography of laser hardened F1140 steel specimen showing a thin external decarburized layer. 
TABLA IV.- Comparación de los resultados experimentales y calculados del temple superficial de acero F1140 con un haz láser de $\mathrm{CO}_{2}$ con una distribución de intensidad transversal $\mathrm{TEM}_{01} * \mathrm{y}$ una potencia de $1.300 \mathrm{~W}$

TABLE IV.- Comparison of predicted and experimental results for a $\mathrm{CO}_{2}$ laser surface hardening of F1140 steel (TEM $01 *$ transversal intensity distribution and $P=1300 \mathrm{~W}$ )

\begin{tabular}{|c|c|c|c|}
\hline Parámetro & Medido & Calculado & Error relativo \\
\hline $\mathrm{y}_{1}(\mathrm{~mm})$ & $6,50 \pm 0,01$ & 6,20 & $-4,62 \%$ \\
$\mathrm{z}_{1}(\mathrm{~mm})$ & $0,98 \pm 0,01$ & 1,10 & $+12,24 \%$ \\
$\mathrm{z}_{3}(\mathrm{~mm})$ & $0,80 \pm 0,01$ & 0,85 & $+6,25 \%$ \\
\hline
\end{tabular}

consecuencia de la dificultad de los experimentadores de predecir la temperatura máxima alcanzada por el vidrio durante la aplicación rutinaria de dicha técnica. La aplicación TEMPLUM para resolver el problema es bastante directa con la ayuda de las rutinas de pre-procesado y las nuevas rutinas incluidas en el código (especialmente adecuadas para la resolución de este tipo específico de problemas) y facilita los resultados deseados de una manera adecuada para su comparación con resultados experimentales.

En la figura 14 (superior) se muestra un mapa térmico superficial de la pieza tratada en la que las isotermas encierran las zonas que han alcanzado la temperatura suficiente para fundir (ablandar) el vidrio y que se correlacionan con las zonas pulidas, una vez enfriadas, en el correspondiente caso experimental mostrado en la figura 14 (inferior).

\section{CONCLUSIONES}

En vista de los resultados presentados y las propiedades de convergencia, estabilidad y precisión evaluadas específicamente en trabajos previos (9), tiene que considerarse el código TEMPLUM como una valiosa herramienta de simulación numérica comparable con los códigos más avanzados desarrollados para la predicción del comportamiento dinámico de los campos térmicos inducidos en materiales mediante fuentes de calor externas.

Adicionalmente, y provisto de un conjunto de rutinas específicas añadidas al código numérico base con el fin de considerar las características particulares de las aplicaciones del tratamiento térmico superficial de materiales en fase condensada con láser, incluyendo las propiedades térmicas y ópticas del material en función de la temperatura y las condiciones de contornos propias de los procesos, el

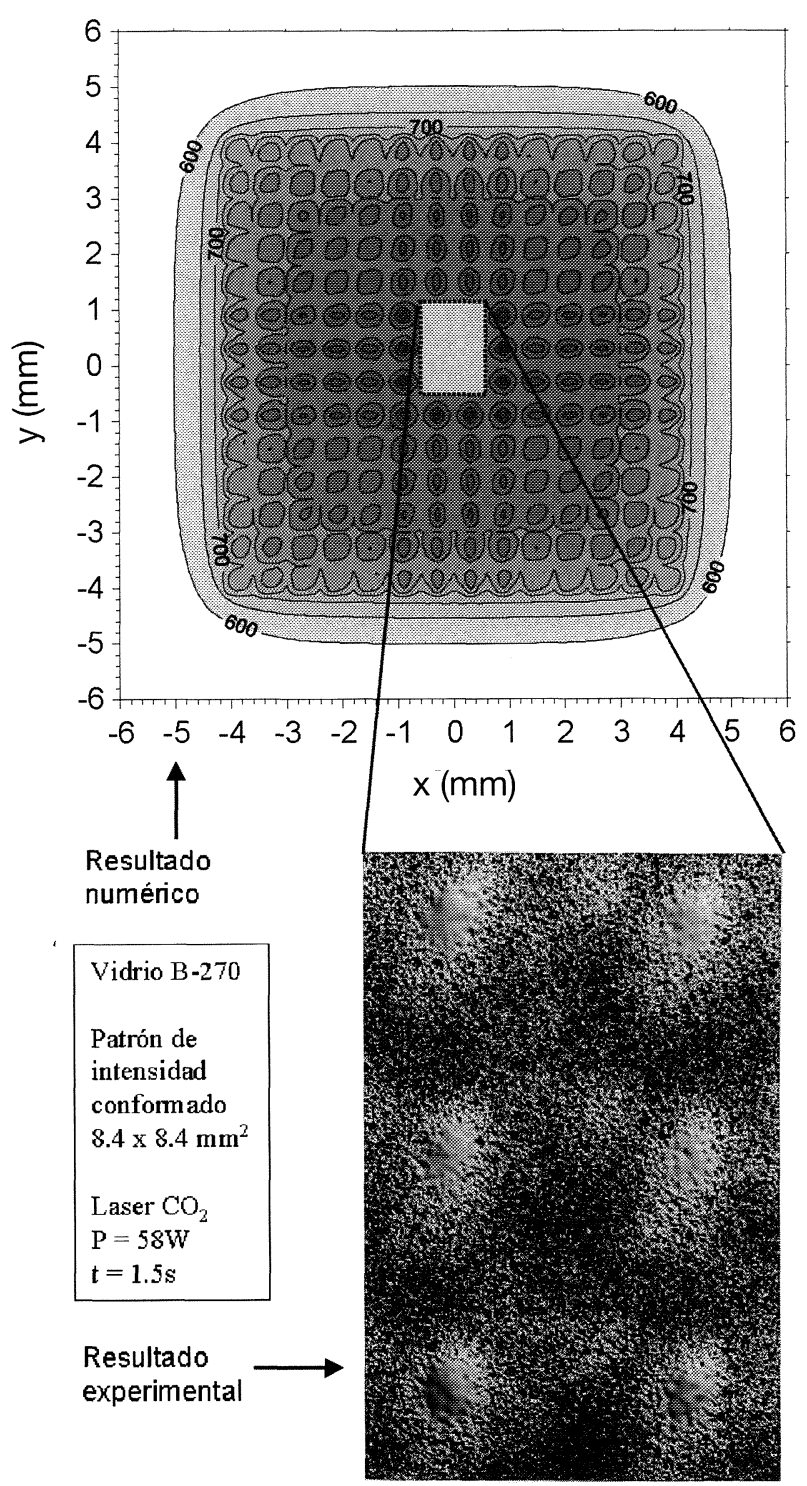

Fig. 14.- Mapa térmico superficial de vidrio B-270 al final de la incidencia que muestra la predicción de las zonas tratadas (superior) y fotografía de la superficie del vidrio B-270 que muestra las zonas pulidas después del tratamiento láser (inferior).

FIG. 14.-B270 optical glass predicted surface thermal contours (top) and B270 optical glass surface photograph (bottom) showing the polished areas after the laser treatment.

código TEMPLUM tiene que ser considerado como una herramienta realmente útil para la predicción y el análisis de las aplicaciones de tratamiento térmico superficial en una amplia variedad de procesos industriales.

\section{Lista de símbolos}

$\begin{array}{lll}\alpha & \text { Absortividad } & \left(\mathrm{m}^{-1}\right) \\ \kappa & \text { Conductividad térmica } & \left(\mathrm{W} \mathrm{m}^{-1}{ }^{\circ} \mathrm{C}^{-1}\right)\end{array}$ 
$\lambda \quad$ Longitud de onda de la ra-

(m) diación electromagnética

$\rho \quad$ Densidad másica

A Absortancia o fracción ab-

$\left(\mathrm{kg} \mathrm{m}^{-3}\right)$

(Adim.) sorbida de la intensidad incidente

$C_{\mathrm{p}} \quad$ Capacidad calorífica a pre-

$\left(\mathrm{J} \mathrm{kg}^{-1}{ }^{\circ} \mathrm{C}^{-1}\right)$ sión constante

$d \quad$ Espesor de una placa

(m)

E Energía absorbida por uni-

$\left(\mathrm{W} \mathrm{m}^{-3}\right)$ dad de volumen

$I_{0} \quad$ Intensidad en el centro de una distribución gaussiana o de simetría circular

$P \quad$ Potencia

$r_{\mathrm{C}} \quad$ Radio característico del modo transversal $\mathrm{TEM}_{01}$ *

$t \quad$ Tiempo

$\left(\mathrm{W} \mathrm{m}^{-2}\right)$

$t_{0} \quad$ Instante inicial

$t_{\mathrm{L}} \quad$ Duración de un pulso láser

$T_{0} \quad$ Temperatura inicial

(K)

$T_{\mathrm{f}} \quad$ Temperatura ambiente
Traitements des Matériaux, Ecole de Printemps, Sirueil, (Francia), 1991: 1-26.

(2) Ion, J.C.: "Modelling of Laser Material Processing". The Industrial Laser Annual Handbook. D. Belforte \& M. Levitt Eds. Penn Well Books, 1992: 39-47.

(3) AMEs, W.F.: "Numerical Methods for Partial Differential Equations". $2^{\text {nd }}$ Ed. Academic Press, 1977.

(4) LAPIDUs, L., Pinder, G.F.: "Numerical Solution of Partial Differential Equiations in Science and -Engineering". John Wiley and sons, 1982.

(5) Mazumder, J., Steen, W.M.: Appl. Phys, 51, 1980: 941947.

(6) Fritsch, H.U., BergmanN, H.W.: "Influence of the Carbon Diffusion During Laser Transformation Hardening. Numerical Simulation and Experimental Verification". Memorandum interno, Universidad de Erlagen, Nuremberg (Alemania), 1982.

(7) Kechemair, D.: "Instrumentation et Optimization de la Trempe Superficielle des Aciers par Laser $\mathrm{CO}_{2}$ Continu”. Tesis Doctoral. Université de Paris-Sud, (Francia), 1989.

(8) Galantucci, L., Tricarico, L. "Transient Numerical Analysis of Laser Heat Treatment Using FEM", Proc. LANE'94, Vol. I. Ed. M. Geiger y F. Vollertsen, Meisenbach Bamberg, 1994: 295-316.

(9) Garcia-Beltran, A.: "Desarrollo y Validación de un Modelo Computacional para la Predicción y Caracterización de Procesos de Tratamiento Térmico Superficial de Materiales con Láser". Tesis Doctoral. Universidad Politécnica de Madrid (España), 1996.

(10) Shapiro, A.B., Edwards, A.L.: "TOPAZ2D Heat Transfer Code Users Manual and Thermal Property Data Base". Report UCRL-ID-104558 (rev. 1.), Lawrence Livermore National Laboratory, University of California (EE. UU.), 1990.

(11) ShAPIRo, A.B.: "TOPAZ3D - A Three Dimensional Finite Element Heat Transfer Code". Report UCID-20484, Lawrence Livermore National Laboratory University of California (EE. UU.)1985:

(12) Laguarta, F. et al.: Appl. Optics, 33, 1994: 6508-6513.

(13) OcaÑA, J.L. et al:: "Analysis of the Effect of Optically induced Local Non-Uniformities in Laser Surface Treatment Applications". Proc. of 1996 Conference on Lasers and Electro-Optics Europe. CLEO/EQEC, 1996: 227. 